\title{
CIÊNCIAS AMBIENTAIS
}

\section{Caracterização espacial e análise da qualidade da água da Bacia do Arroio Moreira/Fragata, Pelotas, RS}

\author{
Spatial characterization and analysis of water quality in the basin \\ of the Stream Moreira/Fragata, RS
}

Lidiane Schmalfuss Valadão ${ }^{1}$; Régis da Silva Pereira ${ }^{1}$; Marcelo Dutra da Silva²; Jander Luis Fernandes Monks ${ }^{1}$

\begin{abstract}
RESUMO
A urbanização desordenada do solo e as ações antrópicas causam impactos negativos em função da degradação da área e qualidade da água, induzindo a poluição do corpo hídrico, já que a procura pelos recursos excede a disponibilidade. Assim, o objetivo deste trabalho é caracterizar a paisagem e avaliar a qualidade da água da Bacia do Arroio Moreira/Fragata, determinando os fatores que comprometem a área, pois esta é responsável por parte do abastecimento do município de Pelotas/RS. Os parâmetros físico-químicos ( $\mathrm{pH}$, condutividade, turbidez, oxigênio dissolvido, DBO5, cloretos, nitrogênio e fósforo totais) e biológicos (coliformes totais e termotolerantes) foram avaliados durante o período de novembro de 2015 a junho de 2016, em seis pontos distribuídos ao longo dos tributários da bacia. Ao realizar a comparação individual dos valores obtidos com as diretrizes da resolução CONAMA n $357 / 2005$, para água doce classe 2, verificou-se que a porção sul da bacia é mais crítica para a maioria dos parâmetros. Isto pode estar relacionado ao fato desta região possuir maior percentual de urbanização e concentração de indústrias alimentícias, que contribuem com efluentes doméstico e agroindustrial.
\end{abstract}

Palavras-chave: Equilíbrio aquático, Urbanização, Análise métrica.

\begin{abstract}
The disorderly urbanization of the soil and the anthropic actions cause negative impacts due to the degradation of the area and quality of the water, inducing the pollution of the water body, since the demand for the resources exceeds the availability. Thus, the objective of this work is to characterize the landscape and evaluate the water quality of the Stream Moreira/Fragata Basin, determining the factors that compromise the area, since this is responsible for part of the supply of the municipality of Pelotas/RS. The physical-chemical parameters ( $\mathrm{pH}$, conductivity, turbidity, dissolved oxygen, DBO5, total chlorides, nitrogen and phosphorus) and biological parameters (total and thermotolerant coliforms) were evaluated during the period from November 2015 to June 2016, in six points distributed along the tributaries of the basin. When comparing the values obtained with the guidelines of CONAMA Resolution No. 357/2005 for freshwater class 2, it was verified that the southern portion of the basin is more critical for most of the parameters. This may be related to the fact that this region has a higher percentage of urbanization and concentration of food industries, which contribute to domestic and agroindustrial effluents.
\end{abstract}

Keywords: Aquatic balance, Urbanization, Metric analysis.

\footnotetext{
${ }^{1}$ IFSul - Instituto Federal de Educação, Ciência e Tecnologia Sul-rio-grandense, Pelotas/RS - Brasil.

2 FURG - Universidade Federal do Rio Grande - Rio Grande/RS - Brasil.
} 


\section{INTRODUÇÃO}

A água é um recurso natural renovável indispensável para a manutenção das diversas formas de vida, para isso é importante que suas propriedades físico-químicas e biológicas estejam equilibradas (ARAIN et al., 2014; GOMES SILVA et al., 2014; BEZERRA et al., 2013).

A qualidade da água está diretamente relacionada com o uso e ocupação do solo. A ocupação irregular causa impactos negativos, como: mudanças nos cursos, nas características físico-químicas naturais da água, cobertura da terra e sobre a biota existente (ARAIN et al., 2014; ROCHA et al., 2015).

Segundo Arpine \& Gayane (2016) e Netto et al. (2013), a urbanização desordenada ocasiona o uso indisciplinado dos corpos hídricos, provocando a degradação ambiental, afetando o desenvolvimento econômico e social, a saúde humana, o equilíbrio dos ecossistemas aquáticos e interferindo principalmente no aproveitamento das águas superficiais, devido à poluição.

Em conformidade com o Art. 13, § $1^{\circ}$ do Decreto n 73.030/1973 (BRASIL, 1973), define-se poluição das águas como qualquer alteração de suas propriedades físicas, químicas ou biológicas, que possa importar em prejuízo à saúde, à segurança e ao bem-estar das populações, causar dano à flora e à fauna, ou comprometer o seu uso para fins sociais e econômicos.

Mohamed et al. (2015) e Rocha et al. (2015) citam que os impactos negativos ao se tratar de qualidade da água, podem ser de origem pontual, como: disposição inadequada de resíduos sólidos, desmatamentos, erosão, descargas de efluentes domésticos e industriais; ou difusa, (pecuária, lixiviação de fertilizantes e pesticidas provenientes de áreas agrícolas).

A liberação de compostos químicos confere um certo grau de poluição ao corpo hídrico e como resultado há formação de misturas complexas potencialmente perigosas no ambiente aquático, gerando o aumento das concentrações dos parâmetros de qualidade (KUZMANOVIĆ et al., 2016). Além disto, a avaliação dos parâmetros biológicos e físico-químicos da água permitem detectar as possíveis influências antropogênicas, industriais e agrárias sob a qualidade dos corpos hídricos (MWANAMOKI et al., 2014).

As informaç̃os de qualidade de água são complexas, pois diferentes substâncias podem intervir nas propriedades naturais, dependendo das suas concentrações, usos da água, condições hidrológicas, características biofísicas, geologia natural e fontes de poluição (PEREIRA, 2004; TABARI et al., 2011). Porém, os teores das substâncias flutuam ao longo do ano, seja acima ou abaixo dos níveis que podem ser prejudiciais, (ARPINE \& GAYANE, 2016). Por conseguinte, torna-se imprescindível estabelecer padrões de qualidade para cada atividade, a fim de manter as características de natureza física, química e biológica, evitando danos ao meio ambiente e saúde pública (BETEMPS et al., 2014).

Diante deste contexto, apesar da importância socioeconômica da Bacia do Arroio Moreira/Fragata, que compõe um dos três mananciais de abastecimento de água da cidade de Pelotas/RS - a estação de tratamento de água do Moreira, (SANEP, 2016) - a mesma vem sofrendo com a ocupação de extensas áreas no decorrer do seu espaço natural, alterando a qualidade da água e modificando a paisagem do leito do recurso hídrico. 
Desta forma, o objetivo deste trabalho foi caracterizar e avaliar a qualidade da água da Bacia do Arroio Moreira/Fragata, no quadro da organização espacial dominante, visando determinar os fatores que comprometem o ambiente em estudo.

\section{METODOLOGIA}

\subsection{Descrição da área de estudo}

A bacia do Arroio Moreira/Fragata, encontra-se inserida na grande Bacia Litoral 40, do sistema hidrográfico do estado do Rio Grande do Sul (L40-RS), sendo uma das vinte e seis bacias do complexo hídrico do estado, situando-se no extremo sul (NETO, 2009). Localizada no sudoeste do município de Pelotas/RS, a bacia abrange área de, aproximadamente, 87 km² (SOUSA, 2008 apud HANSMANN, 2013). De acordo com o plano ambiental de Pelotas (PELOTAS, 2013), a mesma possui extensas áreas de banhados e importantes areais.

A distribuição da bacia compreende os municípios de Pelotas, Capão do Leão e Morro Redondo, além dos Distritos da Cascata, do Monte Bonito e da Área Urbana, conforme a figura 1. À margem direita, uma pequena porção, incluindo a Lagoa do Fragata, está situada no município do Capão do Leão. 0 Arroio Moreira/Fragata, rio principal, apresenta seu desnível geométrico de 285 m, percorrendo 37,9 km de extensão, desembocando na Lagoa do Fragata. Na sua margem esquerda, encontram-se os arroios Pestana, Michaela e Moinho e na margem direita está o arroio Taquara (NETO, 2009; PELOTAS, 2013).

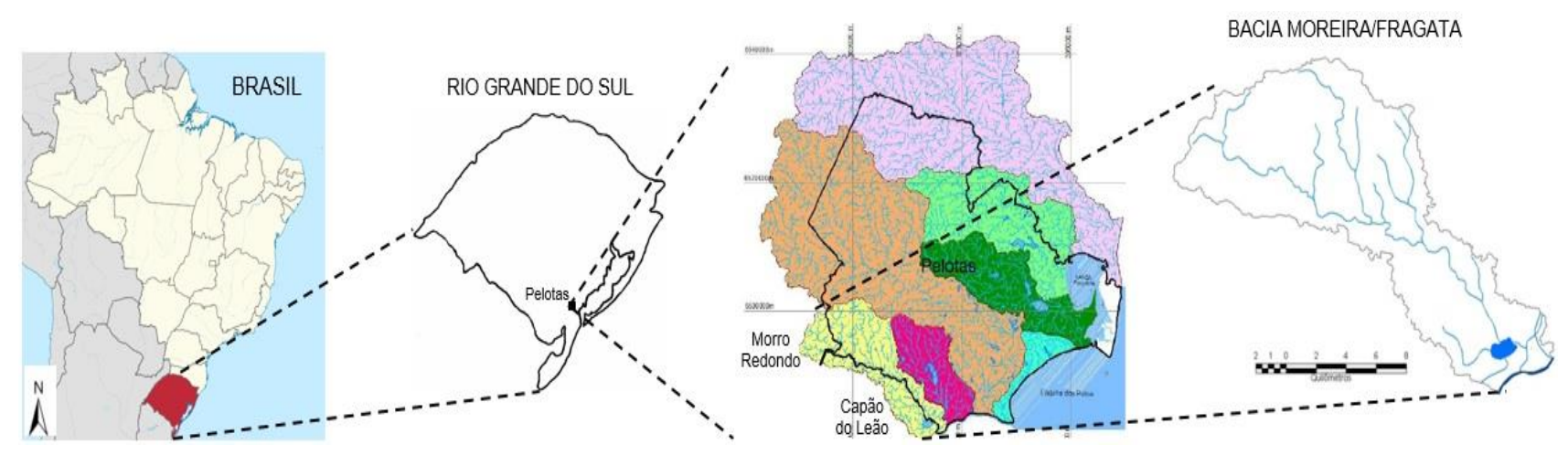

Figura 1. Mapa de localização da Bacia do Arroio Moreira/Fragata.

\subsection{Caracterização espacial}

Para caracterizar o espaço delimitado pela Bacia do Arroio Moreira/Fragata o trabalho fez uso de uma cena de satélite LandSat 8, de fevereiro de 2016, obtida pelo Earth Explore, disponível no sistema Unites States Geological Survey-USGS e de ferramentas de análise espacial (métricas de ecologia de paisagem) para ilustrar o arranjo da bacia, que compreende ambientes distintos, de predomínio florestal, nos terrenos mais dobrados e elevados, junto das cabeceiras; e de áreas úmidas de planície, no baixo curso da bacia, no vale do Canal São Gonçalo.

Foram utilizados os softwares QGIS 2.8 para a reprojeção, mosaico de bandas e recorte da cena pela máscara (limite da bacia); o SPRING 5.4 para a segmentação, treinamento de coleta de amostras e 
classificação do recorte da imagem; e o ArcGIS 10 para a análise métrica do cenário da bacia, a partir do Plugin Patch Analyst, onde foram trabalhadas as métricas de área, borda, forma e diversidade.

\subsection{Obtenção de amostras}

A situação ambiental do ecossistema foi avaliada com o uso de parâmetros físico-químicos e microbiológicos da água. A amostragem foi realizada em quatro campanhas de coleta (novembro de 2015; e janeiro, abril e junho de 2016), período de maior probabilidade de contribuição por lançamentos, devido as atividades agroindustriais e agrícolas serem mais intensas.

O comportamento espacial dos indicadores de qualidade foi avaliado através do georreferenciamento dos locais de amostragem (tabela 1), com o auxílio do GPS modelo GARMIN Etrex Vita $\mathrm{H}$, subsidiando indicar os possíveis pontos de entrada de contaminantes no recurso hídrico.

Tabela 1. Georreferenciamento dos locais de amostragem de água ao longo da Bacia do Arroio Moreira/Fragata.

\begin{tabular}{cccc}
\hline Local & Identificação do local & Coordenada S & $\begin{array}{c}\text { Coordenada } \\
\mathbf{0}\end{array}$ \\
\hline P1 & Jusante a empresa de laticínios (baixo curso) & $31^{\circ} 45^{\prime} 59.97^{\prime \prime}$ & $52^{\circ} 23^{\prime} 50.32^{\prime \prime}$ \\
P2 & Montante a empresa de laticínios e Jusante a indústria & $31^{\circ} 45^{\prime} 38.93^{\prime \prime}$ & $52^{\circ} 24^{\prime} 09.49^{\prime \prime}$ \\
de conservas (baixo curso) & & & \\
P3 & Jusante a indústria frigorífica (baixo curso) & $31^{\circ} 44^{\prime} 56.39^{\prime \prime}$ & $52^{\circ} 24^{\prime} 52.13^{\prime \prime}$ \\
P4 & Ponte sobre o Arroio Pestanas (médio curso) & $31^{\circ} 42^{\prime} 50.55^{\prime \prime}$ & $52^{\circ} 28^{\prime} 36.46^{\prime \prime}$ \\
P5 & Montante da estação de tratamento de água (alto & $31^{\circ} 42^{\prime} 03.47^{\prime \prime}$ & $52^{\circ} 30^{\prime} 55.44^{\prime \prime}$ \\
P6 & Jusante a indústria de conservas (alto curso) & $31^{\circ} 40^{\prime} 23.56^{\prime \prime}$ & $52^{\circ} 30^{\prime} 04.16^{\prime \prime}$ \\
\hline
\end{tabular}

As coletas das amostras pontuais de água, foram obtidas em seis pontos distribuídos do baixo ao alto curso da Bacia Arroio Moreira/Fragata, acompanhando seus tributários junto às zonas urbana e rural, dos municípios de Pelotas e Capão do Leão, conforme a figura 2.

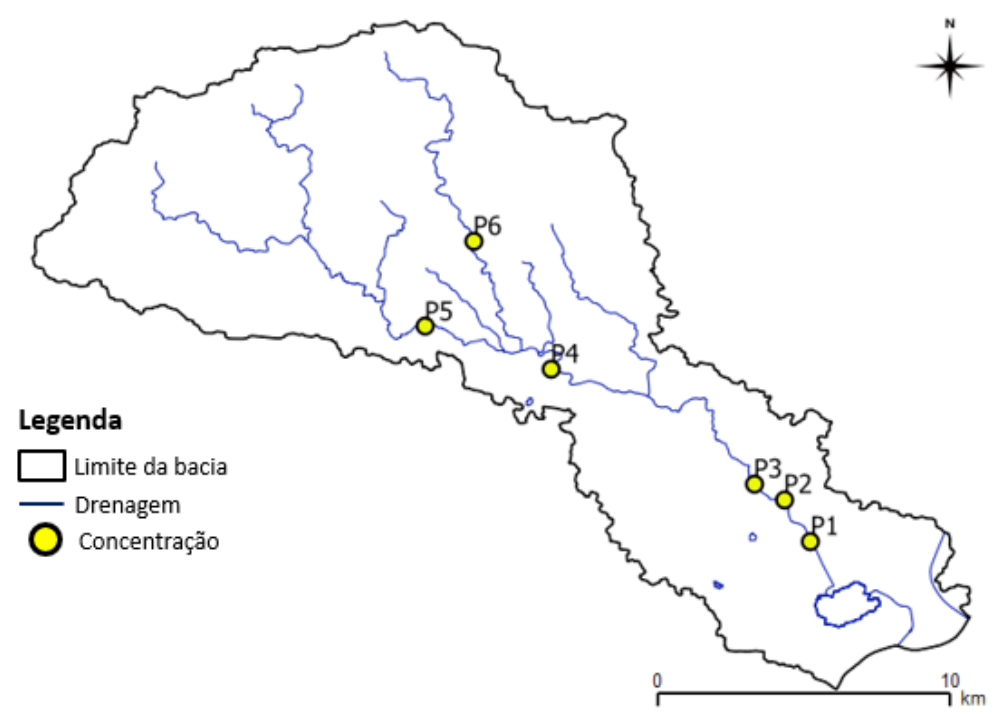

Figura 2. Localização dos pontos georreferenciados, na Bacia do Arroio Moreira/Fragata. 
A amostragem da água para os parâmetros físico-químicos nos pontos P1, P2 e P3, foi composta, de modo a atender as necessidades destes locais, ou seja, em três pontos de coleta, assim distribuídos: $25 \%$ da largura do arroio a meia profundidade; $50 \%$ da largura do arroio a $50 \%$ de profundidade; e 75\% da largura do arroio a meia profundidade (NBR 9897, 1987). Enquanto que nos pontos P4, P5 e P6, a coleta foi caracterizada como simples, no ponto central. A amostragem para análise microbiológica foi simples e realizada a cerca de $20 \mathrm{~cm}$ da superfície e em contracorrente, em todos os pontos.

Todas as amostras foram armazenadas e conservadas em frascos de polietileno, conforme o caso específico (NBR 9898, 1987), exceto a amostra para análise microbiológica, a qual foi retida em frasco de borosilicato esterilizado. Logo, ambos foram armazenados sob refrigeração à $\pm 4^{\circ} \mathrm{C}$ e transportados para o laboratório.

\subsection{Análises da água}

A tabela 2, apresenta os parâmetros físico-químicos avaliados nas amostras de água e as respectivas metodologias utilizadas. Todos as análises foram determinadas em triplicata.

Tabela 2. Parâmetros físico-químicos analisados nas amostras de água da Bacia do Arroio Moreira/Fragata.

\begin{tabular}{|c|c|}
\hline Análise & Metodologia \\
\hline Potencial hidrogênionico (pH) & pHmetro Marconi modelo MA 522/P \\
\hline Oxigênio dissolvido (OD) & Oxímetro HANNA Instruments modelo HI 9146-04 \\
\hline Condutividade elétrica & Condutivímetro Instrutherm modelo CD-830 \\
\hline Turbidez & Turbidimetro Tecnopon modelo TB-1000 \\
\hline Demanda biológica de oxigênio (DBO5) & APHA, 2005 \\
\hline Cloretos & APHA, 2005 \\
\hline Fósforo total & $\begin{array}{l}\text { Multiparameter Bench Photometer, marca Hanna: } \\
\text { métodos com ácido ascórbico (APHA, 2005) }\end{array}$ \\
\hline Nitrogênio total & $\begin{array}{l}\text { Multiparameter Bench Photometer, marca Hanna: } \\
\text { métodos com ácido cromatrópico (APHA, 2005) }\end{array}$ \\
\hline
\end{tabular}

Para avaliação da contaminação microbiológica da água, determinou-se os coliformes totais e termotolerantes/fecais. O teste presuntivo para coliformes foi realizado utilizando o caldo lauril sulfato triptose (LST) e os testes confirmativos para coliformes totais e termotolerantes, em meio do caldo lactosado bile verde brilhante (VBBL) e caldo $\mathrm{EC}$, respectivamente. Utilizou-se a técnica do número mais provável (BRASIL, 2006).

\section{RESULTADOS E DISCUSSÃO}

A classificação espacial da área da Bacia do Arroio Moreira/Fragata (figura 3), permite afirmar que o local se encontra fragmentado, ou seja, com diferentes e pequenas manchas isoladas. Segundo Cerqueira et al. (2003), a fragmentação antrópica de habitats transforma a paisagem em manchas isoladas do estado original em meio às plantações e áreas urbanas, tal fato tem ocasionado impactos diretos sob a flora e, consequentemente, no habitat da fauna. 


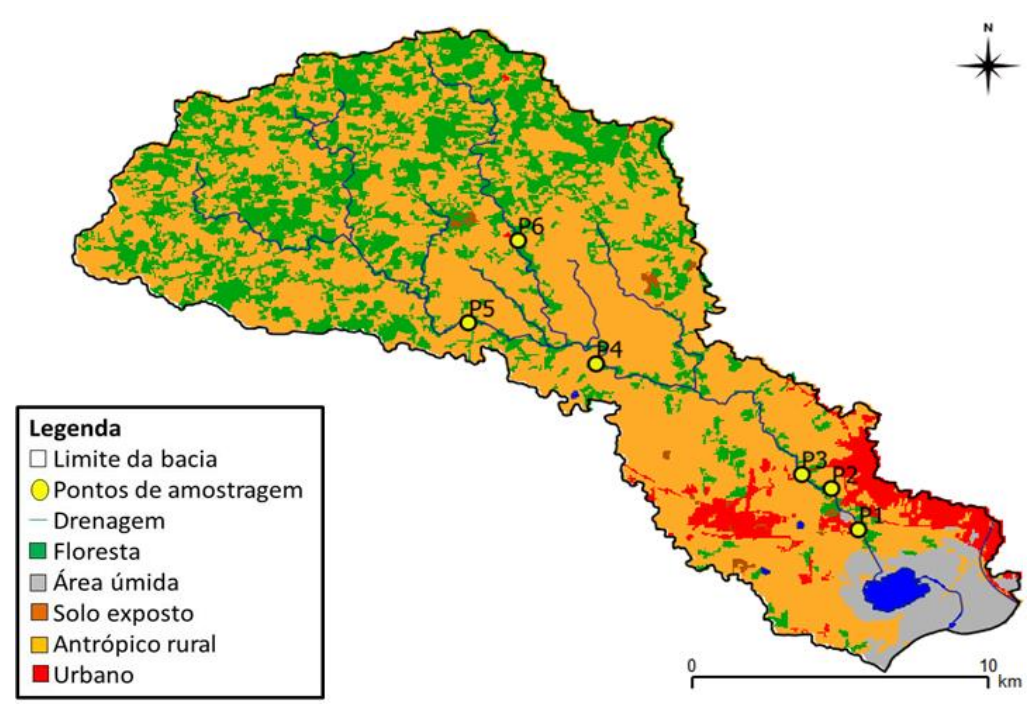

Figura 3. Caracterização espacial da Bacia do Arroio Moreira/Fragata.

Através da análise métrica da paisagem, obteve-se os planos de classe e de paisagem, conforme a tabela 2. Ao considerar o plano de classe, verifica-se que a área de classe (CA) do antrópico rural é maior que a área natural remanescente, isto é, florestas e áreas úmidas, o que indica a existência de ocupação de áreas naturais na delimitação da bacia. Contudo, a extensão total da borda (TE) sugere que a classe de florestas é altamente passível de sofrer retalhamento e divisão, já que esta é acompanhada de um grande número de manchas (NumP). Estas circunstâncias promovem impactos negativos, devido as alterações dos cursos hídricos, ocasionando rebaixamento do lençol freático, influenciando diretamente no equilíbrio hídrico e alterando a qualidade da água dos tributários da Bacia do Arroio Moreira/Fragata.

A forma é a relação entre a área e a borda de cada classe, onde os valores obtidos (tabela 2), demonstram que as florestas se aproximam de um $(1,00)$, sendo consideradas mais arredondadas e consequentemente mais indicadas para a conservação dos fragmentos (SILVA \& SOUZA, 2014).

Na análise do plano de paisagem, (tabela 2), os valores revelam ser este um cenário diverso, bastante heterogêneo e pouco uniforme, ou seja, tem muitos elementos de várias classes e a quantidade e tamanho dos elementos não segue um padrão de uniformidade, conforme os índices de diversidade (SDI) e uniformidade de Shannon (SEI). Desta forma, torna o local desfavorável a diversidade de espécies, sendo necessário empregar um planejamento e gestão ambiental, através dos órgãos públicos, para a preservação da qualidade da água e dos habitats naturais remanescentes.

Tabela 2. Plano de classe obtido na análise métrica da bacia do Arroio Moreira/Fragata, valores em hectares (ha).

\begin{tabular}{|c|c|c|c|c|c|c|c|c|c|c|}
\hline \multicolumn{11}{|c|}{ ANÁLISE NO PLANO DE CLASSE } \\
\hline \multirow{2}{*}{$\operatorname{Cod}^{1}$} & \multirow{2}{*}{ Classe } & \multirow{2}{*}{ NumP2 } & \multicolumn{3}{|c|}{ Área } & \multicolumn{3}{|c|}{ Borda } & \multicolumn{2}{|c|}{ Forma } \\
\hline & & & $\mathbf{C A}^{3}$ & MPS $^{4}$ & PSSD $^{5}$ & TE $^{6}$ & MPE $^{7}$ & $\mathrm{ED}^{8}$ & MSI $^{9}$ & MPFD $^{10}$ \\
\hline 1 & Floresta & 462 & 5965.83 & 12.91 & 59.30 & 1139936.27 & 2467.39 & 16.44 & 0.71 & 1.19 \\
\hline 2 & Antrópico rural & 230 & 14356.98 & 62.42 & 866.63 & 1360134.20 & 5913.63 & 19.61 & 0.57 & 1.18 \\
\hline 3 & Solo exposto & 34 & 106.27 & 3.13 & 5.38 & 28760.55 & 845.90 & 0.41 & 0.50 & 1.18 \\
\hline 4 & Urbano & 96 & 1107.47 & 11.54 & 69.38 & 146259.37 & 1523.54 & 2.11 & 0.54 & 1.19 \\
\hline 5 & Água & 14 & 186.96 & 13.35 & 43.00 & 14500.11 & 1035.72 & 0.21 & 0.40 & 1.17 \\
\hline 6 & Áreas úmidas & 16 & 1080.18 & 67.51 & 246.62 & 72680.30 & 4542.52 & 1.05 & 0.60 & 1.18 \\
\hline
\end{tabular}




\begin{tabular}{|c|c|c|c|c|c|c|c|c|c|c|c|c|}
\hline \multicolumn{13}{|c|}{ ANÁLISE NO PLANO DE PAISAGEM } \\
\hline \multirow{2}{*}{$\operatorname{Cod}^{1}$} & \multirow{2}{*}{ Classe } & \multirow{2}{*}{ NumP2 } & \multicolumn{3}{|c|}{ Área } & \multicolumn{3}{|c|}{ Borda } & \multicolumn{2}{|c|}{ Forma } & \multicolumn{2}{|c|}{ Diversidade } \\
\hline & & & MPS $^{4}$ & PSSD $^{5}$ & TLA $^{11}$ & $\mathrm{TE}^{6}$ & MPE7 $^{7}$ & $\mathrm{ED}^{8}$ & MSI $^{9}$ & MPFD $^{10}$ & SDI $^{12}$ & SEI $^{13}$ \\
\hline All & Paisagem & 853 & 81.30 & 1655.34 & 69345.05 & 3000973.92 & 3518.14 & 43.28 & 0.64 & 1.19 & 0.96 & 0.49 \\
\hline
\end{tabular}

${ }^{1}$ Cod: Código; ${ }^{2}$ NumP: Número de manchas; ${ }^{3} \mathrm{CA}:$ Área da classe; ${ }^{4} \mathrm{MPS}:$ Tamanho médio das manchas; ${ }^{5} \mathrm{PSSD}$ : Desvio padrão do tamanho das manchas; ${ }^{6} \mathrm{TE}$ : Total de borda; ${ }^{7}$ MPE: Média das bordas das manchas; ${ }^{8} \mathrm{ED}$ : Densidade de borda; ${ }^{9} \mathrm{MSI}$ : Média do índice de forma; ${ }^{10} \mathrm{MPFD}$ : Média da dimensão fractal das manchas; ${ }^{11} \mathrm{TLA}$ : Área de todas as manchas; ${ }^{12} \mathrm{SDI}$ : Índice de diversidade de Shannon; ${ }^{13} \mathrm{SEI}$ : Índice de uniformidade de Shannon.

De acordo com a classificação do CONAMA n 357/2005 (BRASIL, 2005), a bacia do Arroio Moreira/Fragata se enquadra como água doce classe 2, onde o corpo hídrico serve como fonte de abastecimento para consumo humano após tratamento convencional, à recreação de contato primário (presença de campings), à irrigação, a proteção da vida aquática, a aquicultura e atividade de pesca.

Os resultados obtidos para os parâmetros físico-químicos e seus respectivos desvios padrão estão representados na tabela 3. Quando comparados individualmente os parâmetros de condutividade e nitrogênio total com a resolução do CONAMA n 357/2005 (BRASIL, 2005), verifica-se que não há alusão para os mesmos.

No entanto, ao tratar-se da determinação de condutividade, ou seja, relação com o grau de contaminação do corpo hídrico, Saraiva et al. (2009) indica que o valor máximo para água doce não poluída é de $100.00 \mu \mathrm{S} . \mathrm{cm}^{-1}$. Diante dos valores médios apresentados na figura 4, constatou-se que apenas os pontos $\mathrm{P} 1$ e P2, no período de amostragem, apresentam contaminação em relação à presença de sais dissolvidos.

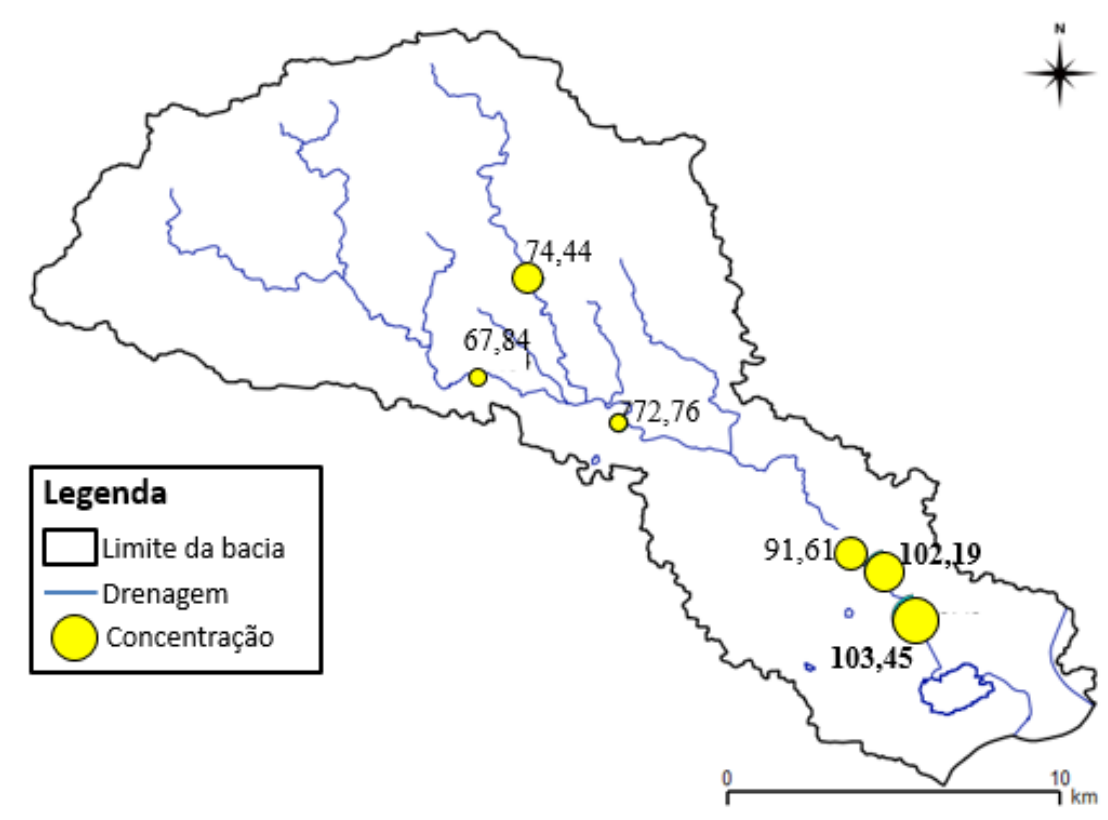

Figura 4. Concentração média de condutividade ( $\mu$ S.cm-1), no período de 2015-2016.

No período estudado, os valores de $\mathrm{pH}$ entre as coletas e os pontos de amostragem, apresentaram variação de $6.58 \pm 0.02$ a $7.41 \pm 0.01$ (tabela 3), mantendo-se próximo da neutralidade e com tendência levemente básica. Valores semelhantes foram encontrados por Valadão et al. (2012), para o canal que recebe a água que desagua da Bacia do Arroio Moreira/Fragata. Ambos os cursos de água apresentaram pH dentro do limite (BRASIL, 2005). Porém, Alberto \& Ribeiro Filho (2012), citam que 
corpos hídricos que apresentam valores para $\mathrm{pH}$ ligeiramente alcalino, indicam influência de despejos de efluentes domésticos, devido a presença de materiais orgânicos.

Segundo Sperling (2005) apud Danelon et al. (2012), a presença de cloretos nos cursos de água é resultante da sua existência natural (dissolução de sais) ou de origem antropogênica (despejos domésticos industriais e águas de irrigação). Conforme observa-se na figura 5, a média dos teores encontrados, está abaixo do máximo permitido pela legislação vigente (BRASIL, 2005) que estabelece $250 \mathrm{mg} \cdot \mathrm{L}^{-1} \mathrm{Cl}^{-1}$. Desta forma, estima-se que a presença de cloretos na bacia seja de origem natural. Todavia, verifica-se através da tabela 3, que as variações mais acentuadas de cloretos, quantitativamente, ocorrem nos pontos P1 e P2, no baixo curso.

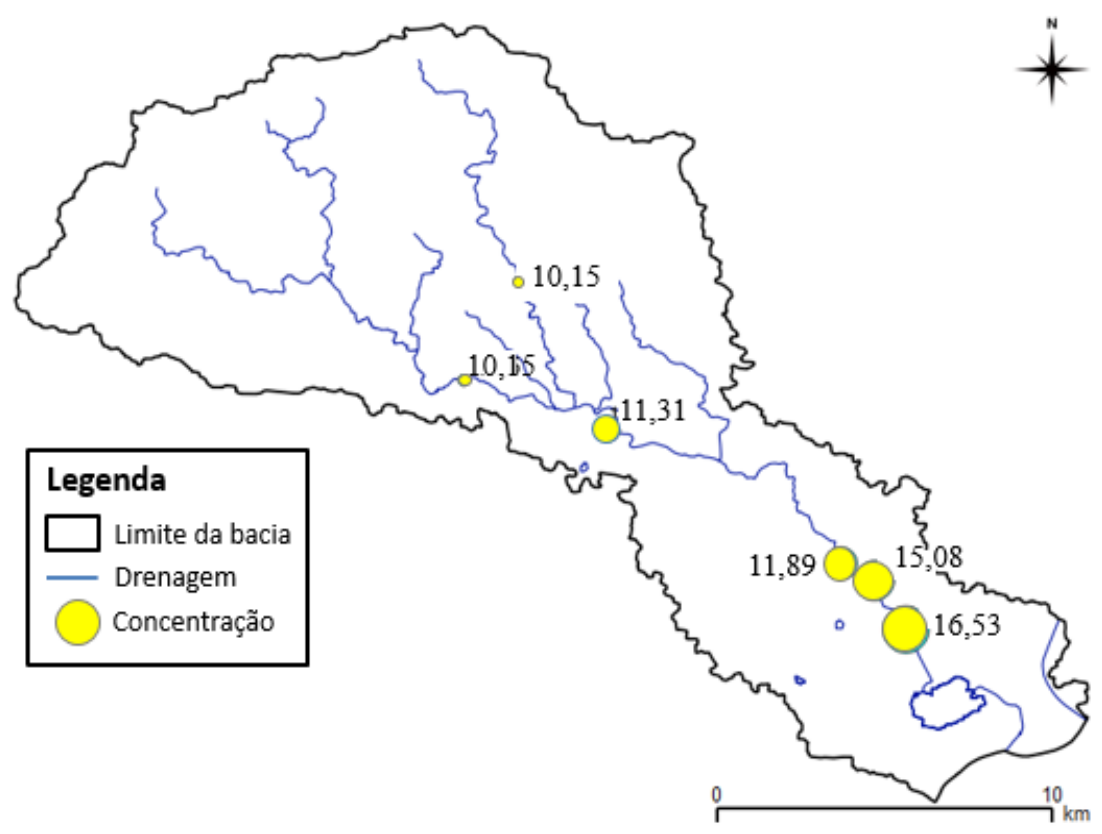

Figura 5. Concentração média de cloretos $\left(\mathrm{mg}_{\mathrm{L}} \mathrm{L}^{-1} \mathrm{Cl}^{-1}\right)$, no período de 2015-2016.

A elevação da turbidez ocasiona modificações nos ciclos biodinâmicos, interferindo na velocidade e intensidade da ação fotossintética (NASCIMENTO et al., 2015). Na tabela 3, verifica-se que no período de estudo, os valores de turbidez variaram de $9.29 \pm 0.13$ a $35.00 \pm 0.00 \mathrm{NTU}$, estando dentro do limite estabelecido pelo CONAMA n 357/2005 (BRASIL, 2005). Tais valores, sofreram elevação quando comparados com os resultados obtidos pelo EPTA, no mesmo período nos anos de 2008/2009, com valores de 2.51 a 13.00 NTU (NETO, 2009).

Os valores médios para a DBO5, expressos na tabela 3, em todos os locais analisados, estão acima do limite estipulado pelo CONAMA n 357/2005 (BRASIL, 2005) de $5.00 \mathrm{mg}^{-\mathrm{L}^{-1}}$, indicando que há entrada de matéria orgânica no arroio. Logo, implica na diminuição da taxa de oxigênio dissolvido na água, principalmente nos pontos P1, P2 e P3, comprometendo a sobrevivência dos peixes e macroinvertebrados (SOUZA \& GASTALDINI, 2014). 
Tabela 3. Valores máximos, mínimos e médios, e seus respectivos desvios padrão dos parâmetros físico-químicos para as amostras de água da Bacia do Arroio Moreira/Fragata, no período de novembro/2015 a junho/2016.

\begin{tabular}{|c|c|c|c|c|c|c|c|c|c|}
\hline \multirow[b]{2}{*}{ Local } & \multirow[b]{2}{*}{ Medida } & \multicolumn{8}{|c|}{ Parâmetro } \\
\hline & & pH & Turbidez (NTU) & Cond $^{1}$ & $\mathbf{O D}^{2}$ & $\begin{array}{c}\mathbf{D B O}^{3} \\
\left(\mathrm{mg} \cdot \mathrm{L}^{-1} \mathrm{O}_{2}\right)\end{array}$ & $\begin{array}{c}\mathbf{N T}^{4} \\
\left(\mathrm{mg} \cdot \mathrm{L}^{-1}\right)\end{array}$ & $\begin{array}{c}\mathbf{P T}^{5} \\
\left(\mathrm{mg} \cdot \mathrm{L}^{-1}\right)\end{array}$ & $\begin{array}{l}\text { Cloretos } \\
\left(\mathrm{mg} \cdot \mathrm{L}^{-1} \mathrm{Cl}^{-}\right)\end{array}$ \\
\hline & Máximo & $7.09 \pm 0.01$ & $29.93 \pm 0.12$ & $122.40 \pm 0.20$ & $12.30 \pm 0.20$ & $22.30 \pm 0.07$ & $19.67 \pm 0.58$ & $0.32 \pm 0.01$ & $22.03 \pm 0.06$ \\
\hline \multirow[t]{3}{*}{$\mathrm{P} 1$} & Mínimo & $6.62 \pm 0.03$ & $14.73 \pm 0.12$ & $87.50 \pm 0.44$ & $2.20 \pm 0.02$ & $7.00 \pm 0.07$ & $2.93 \pm 0.06$ & $0.12 \pm 0.01$ & $13.92 \pm 0.06$ \\
\hline & Média & $6.84 \pm 0.22$ & $21.55 \pm 6.59$ & $103.45 \pm 14.71$ & $7.73 \pm 4.22$ & $12.70 \pm 6.91$ & $12.40 \pm 7.52$ & $0.22 \pm 0.09$ & $16.53 \pm 3.83$ \\
\hline & Máximo & $7.10 \pm 0.02$ & $28.00 \pm 0.00$ & $125.13 \pm 0.15$ & $13.30 \pm 0.01$ & $28.30 \pm 0.14$ & $25.00 \pm 0.00$ & $0.28 \pm 0.01$ & $16.24 \pm 0.06$ \\
\hline \multirow[t]{3}{*}{ P2 } & Mínimo & $6.58 \pm 0.02$ & $14.53 \pm 0.15$ & $87.35 \pm 0.37$ & $3.00 \pm 0.00$ & $5.90 \pm 0.07$ & $0.53 \pm 0.06$ & $0.13 \pm 0.01$ & $12.76 \pm 0.05$ \\
\hline & Média & $6.88 \pm 0.22$ & $20.54 \pm 5.83$ & $102.19 \pm 17.72$ & $9.90 \pm 4.72$ & $12.08 \pm 8.83$ & $11.84 \pm 12.34$ & $0.20 \pm 0.08$ & $15.08 \pm 1.64$ \\
\hline & Máximo & $7.08 \pm 0.02$ & $28.23 \pm 0.40$ & $102.94 \pm 0.10$ & $12.33 \pm 0.21$ & $33.30 \pm 0.00$ & $10.33 \pm 0.58$ & $0.24 \pm 0.01$ & $12.76 \pm 0.06$ \\
\hline \multirow[t]{2}{*}{ P3 } & Mínimo & $6.78 \pm 0.02$ & $14.87 \pm 0.06$ & $78.26 \pm 0.33$ & $7.10 \pm 0.01$ & $4.90 \pm 0.35$ & $10.00 \pm 0.00$ & $0.04 \pm 0.02$ & $11.60 \pm 0.06$ \\
\hline & Média & $6.92 \pm 0.14$ & $21.62 \pm 6.17$ & $91.61 \pm 12.25$ & $9.86 \pm 2.32$ & $12.93 \pm 10.61$ & $10.11 \pm 0.19$ & $0.16 \pm 0.09$ & $11.89 \pm 0.58$ \\
\hline
\end{tabular}




\begin{tabular}{|c|c|c|c|c|c|c|c|c|c|}
\hline & Máximo & $7.41 \pm 0.01$ & $33.67 \pm 0.58$ & $80.00 \pm 0.36$ & $17.80 \pm 0.01$ & $16.80 \pm 0.07$ & $20.67 \pm 0.58$ & $0.27 \pm 0.01$ & $12.76 \pm 0.05$ \\
\hline \multirow[t]{3}{*}{ P4 } & Mínimo & $7.06 \pm 0.01$ & $13.33 \pm 0.12$ & $65.07 \pm 0.78$ & $13.60 \pm 0.02$ & $2.10 \pm 0.07$ & $0.40 \pm 0.00$ & $0.04 \pm 0.01$ & $10.44 \pm 0.00$ \\
\hline & Média & $7.21 \pm 0.15$ & $19.97 \pm 9.33$ & $72.76 \pm 7.28$ & $19.97 \pm 2.15$ & $6.20 \pm 7.08$ & $10.85 \pm 8.34$ & $0.10 \pm 0.11$ & $11.31 \pm 1.11$ \\
\hline & Máximo & $7.31 \pm 0.01$ & $35.00 \pm 0.00$ & $72.62 \pm 0.16$ & $26.73 \pm 0.04$ & $17.30 \pm 0.21$ & $17.67 \pm 0.58$ & $0.17 \pm 0.00$ & $11.60 \pm 0.06$ \\
\hline \multirow[t]{3}{*}{ P5 } & Mínimo & $7.10 \pm 0.01$ & $11.00 \pm 0.00$ & $60.09 \pm 0.50$ & $12.70 \pm 0.01$ & $3.80 \pm 0.07$ & $0.27 \pm 0.06$ & $0.04 \pm 0.01$ & $8.12 \pm 0.04$ \\
\hline & Média & $7.21 \pm 0.09$ & $21.16 \pm 10.33$ & $67.84 \pm 5.60$ & $16.54 \pm 6.81$ & $7.35 \pm 5.64$ & $9.65 \pm 7.15$ & $0.10 \pm 0.06$ & $10.15 \pm 1.46$ \\
\hline & Máximo & $7.39 \pm 0.01$ & $22.07 \pm 0.12$ & $82.33 \pm 0.38$ & $26.73 \pm 0.03$ & $18.40 \pm 0.14$ & $10.33 \pm 0.58$ & $0.06 \pm 0.02$ & $13.92 \pm 0.00$ \\
\hline \multirow[t]{2}{*}{ P6 } & Mínimo & $7.31 \pm 0.09$ & $9.29 \pm 0.13$ & $63.97 \pm 0.28$ & $11.60 \pm 0.02$ & $2.30 \pm 0.14$ & $0.23 \pm 0.06$ & $0.02 \pm 0.01$ & $9.28 \pm 0.06$ \\
\hline & Média & $7.36 \pm 0.03$ & $13.35 \pm 5.88$ & $74.44 \pm 8.81$ & $16.18 \pm 7.07$ & $6.68 \pm 7.83$ & $7.64 \pm 4.94$ & $0.05 \pm 0.02$ & $10.15 \pm 2.90$ \\
\hline \multicolumn{2}{|c|}{$\begin{array}{c}\text { CONAMA } \mathrm{n}^{\circ} \\
357 / 2005\end{array}$} & $6.00-9.00$ & 100.00 & - & $\geq 5.00$ & 5.00 & - & 0.05 & 250.00 \\
\hline
\end{tabular}


Os teores de oxigênio dissolvido (OD) no baixo curso (P1 e P2) são mais críticos, com valores médios de $7.73 \pm 4.22$ e $9.90 \pm 4.72 \mathrm{mg} . \mathrm{L}^{-1}$, respectivamente, apresentando valores mínimos de $2.20 \pm 0.02$ e $3.00 \pm 0.00 \mathrm{mg} \cdot \mathrm{L}^{-1}$ (Tabela 3). Estudos realizados anteriormente pelo EPTA, para os mesmos locais, apresentaram teores médios de $6.24 \mathrm{mg}^{-\mathrm{L}^{-1}}$ (P1) e $7.13 \mathrm{mg} . \mathrm{L}^{-1}$ (P2) (NETO, 2009). A solubilidade de OD na água depende da temperatura e da altitude do local (GOMES SILVA et al., 2014). Desta forma, os baixos valores de OD na água, podem ser explicados pelo período de amostragem apresentar temperaturas mais altas e, principalmente, devido ao aporte de esgoto in natura com alta carga de matéria orgânica biodegradável (SILVA et al., 2014).

O limite de fósforo total estabelecido pela resolução CONAMA no 357/2005 (BRASIL, 2005) para sistemas lóticos (ambiente lótico e tributários de ambientes intermediários) de água doce classe 2 é de $0.05 \mathrm{mg} . \mathrm{L}^{-1}$. A bacia do Arroio Moreira/Fragata superou expressivamente este padrão, alcançando concentrações médias mais elevadas no baixo curso (Figura 6). Os valores médios encontrados pelo EPTA, no mesmo período em 2008/2009, para os seis locais de amostragem deste trabalho, também estavam acima do limite estabelecido (NETO, 2009).

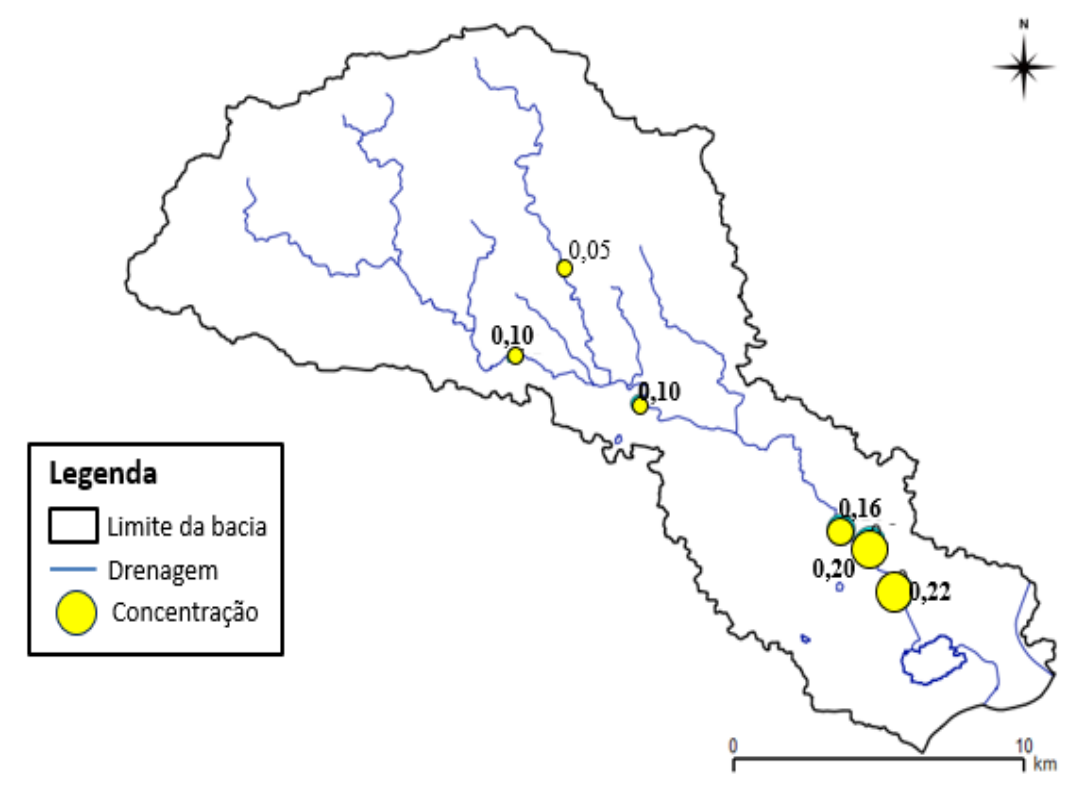

Figura 6. Valores médios de fósforo total $\left(\mathrm{mg} \cdot \mathrm{L}^{-1}\right)$, no período de 2015-2016.

No caso do nitrogênio total, obteve-se valores variando de $0.23 \pm 0.06$ a $25 \pm 0.00 \mathrm{mg} . \mathrm{L}^{-1}$, conforme a tabela 3. Através de comparações realizadas com trabalhos indicados na tabela 4, observa-se que os pesquisadores encontraram valores semelhantes para o nitrogênio total, os quais consideram resultados expressivos indicando diferentes fontes de contaminação.

Tabela 4. Comparação dos resultados com outras referências, concentração mínima e máxima de nitrogênio total em $\mathrm{mg} \cdot \mathrm{L}^{-1}$.

\begin{tabular}{ccccc}
\hline \multirow{2}{*}{ Recurso Hídrico } & \multirow{2}{*}{ Local } & Concentração $\left(\mathbf{m g . L ^ { - 1 }}\right)$ & \multirow{2}{*}{ Referência } \\
\cline { 3 - 3 } & & Nitrogênio Total & \\
\hline Rio Han & Coreia do Sul & 0.00 a 20.00 & & Chang (2008) \\
Igarapé São Francisco & Acre/Brasil & 2.00 a 11.00 & Santi et al. (2012) \\
Rio Taquari & RS/Brasil & 0.52 a 1.12 & & Muller et al. (2016) \\
Arroio Moreira/Fragata & RS/Brasil & 0.50 a 117.00 & & EPTA (NETO, 2009)
\end{tabular}


O teor de nitrogênio associado ao fósforo total pode acarretar o fenômeno de eutrofização, provocando o crescimento desordenado de plantas aquáticas. Essas comprometem os usos múltiplos previstos para as atividades de captação, recreação e preservação da vida aquática aeróbica (BEZERRA et al., 2013). Na área de estudo, as fontes de liberação de nitrogênio para a água da bacia são caracterizadas pelo lançamento de efluente industrial, esgoto sanitário e lixiviação de fertilizantes. Ao tratar-se do fósforo total, a contribuição pode ser ocasionada pela presença de detergentes fosfatados, matéria fecal, drenagem de áreas agrícolas e urbanas, além de efluentes de indústrias de alimentos, laticínios e frigoríficos.

Ao comparar os resultados obtidos nas análises de coliformes totais e termotolerantes (tabela 5) com o limite da Portaria 2.914 do Ministério da Saúde (BRASIL, 2011), verifica-se que todos os pontos de amostragem estão em desacordo, por apresentarem contaminação, tornando a água imprópria para consumo, além de ser fonte de doenças para aqueles que possivelmente realizarem atividades de pesca e banho.

Tabela 5. Resultados de coliformes totais e termotolerantes no período de amostragem na água da Bacia do Arroio Moreira/Fragata.

\begin{tabular}{ccc}
\hline Local & $\begin{array}{c}\text { Coliformes totais } \\
\text { (NTU) }\end{array}$ & $\begin{array}{c}\text { Coliformes termotolerantes } \\
\text { (NTU) }\end{array}$ \\
\hline P1 & $>16$ & $>16$ \\
P2 & $>16$ & $>16$ \\
P3 & $>16$ & $>16$ \\
P4 & $>16$ & $>16$ \\
P5 & $>16$ & $>16$ \\
P6 & $>16$ & $>16$ \\
Portaria & Ausência & Ausência
\end{tabular}

Os coliformes possivelmente são provenientes da atividade de criação de gado na área rural e de esgotos domésticos da área urbana da bacia, apresentando microrganismos patogênicos de origem entérica, animal e humana. (FERREIRA et al., 2015). A contaminação por coliformes fecais se agrava nos meses que correspondem ao período de análise, por ter aumento de temperatura e baixa precipitação de chuvas (EMBRAPA, 2016).

De acordo com os resultados encontrados, neste estudo, verifica-se que o baixo curso da bacia (parte sul) tende a apresentar teores mais elevados para a maioria dos parâmetros, exceto o pH e coliformes termotolerantes que se mantêm ao longo do tributário. Tal fato se justifica, principalmente, porque o baixo curso está mais próximo da área urbanizada, conforme a caracterização espacial (figura 3), a qual não possui, em sua maioria, coleta e tratamento de esgoto adequado. Ainda, há ocorrência de lançamento da carga de resíduos líquidos agroindustriais e contribuição da lixiviação de defensivos agrícolas, que tendem a se concentrar na porção mais baixa, devido a menor declividade.

Segundo o Ministério do Meio Ambiente (MMA, 2016), a parte sul da bacia, região mais afetada, está próxima a uma das áreas prioritárias para conservação da biodiversidade, representada pela área úmida na figura 3, a qual serve de concentração para aves aquáticas e migratórias, sendo importante a conversação deste local. 


\section{CONCLUSÕES}

Verificou-se elevada área de ocupação antrópica no ambiente rural, excedendo a área natural, ocasionando impactos ambientais negativos devido às modificações da paisagem. Através dos resultados encontrados para os parâmetros físico-químicos e microbiológicos, concluiu-se que há alteração na qualidade da água, principalmente na porção sul da bacia, demonstrando a contribuição urbana através da ausência de saneamento básico, despejo de efluentes industriais e lixiviação, que aliada a alteração do regime de escoamento da coluna d'água, contribui para elevação da $\mathrm{DBO}_{5}$, fósforo total e nitrogênio total da água. Deste modo, a aplicação de medidas mitigatórias e políticas públicas, além da conscientização ambiental, devem ser consideradas, visando a proteção e disponibilização dos variados usos da água da Bacia do Arroio Moreira/Fragata.

\section{REFERÊNCIAS}

ABNT. Associação Brasileira de Normas Técnicas. NBR 9897: Planejamento de amostragem de efluentes líquidos e corpos receptores. Rio de Janeiro. 1987. 24 p.

ABNT. Associação Brasileira de Normas Técnicas. NBR 9898: Preservação e técnicas de amostragem de efluentes líquidos e corpos receptores. Rio de Janeiro. 1987. 22 p.

ALBERTO, André; RIBEIRO FILHO, Basílio Gomes. Influência do despejo de esgoto doméstico nas características limnológicas do rio Camandocaia, bacia hidrográfica do rio Piracicaba. Estado de São Paulo. Acta Scientiarum Biological Sciences. v. 34, n. 2, p. 173-179, 2012.

APHA. AMERICAN PUBLIC HEALTH ASSOCIATION. Standard Methods for the Examination of Water and Wastewater, Washington: 21 ed. 1268 p, 2005.

ARAIN, Muhammad Ballal; ULLAH, Ihsan; NIAZ Abdul; SHAH, Nasrullah; SHAH, Afzal; HUSSAIN, Zahid; TARIQ, Muhammad; AFRIDI, Hassan Imran; BAIG. Jameel Ahmad.; KAZI. Tasneem Gul. Evaluation of water quality parameters in drinking water of district Bannu. Pakistan: Multivariate study, Sustainability of Water Quality and Ecology, v. 3, n. 4, p. 114-123, 2014.

ARPINE, Hovhannisyan; GAYANE, Shahnazaryan. Determination of background concentrations of hydrochemical parameters and water quality assessment in the Akhuryan River Basin (Armenia), Physics and Chemistry of the Earth, v. 94, p. 2-9, 2016.

BETEMPS, Glauco Rasmussen; KERSTNER, Talita; SANCHES FILHO, Pedro José. Caracterização físicoquímica da água e determinação de metais pesados ( $\mathrm{Cr}$. $\mathrm{Cu}$. $\mathrm{Pb}$. $\mathrm{Zn}$ ) no sedimento do riacho Arroio do Padre (Arroio do Padre. Brasil/RS), Revista Thema, v. 11, n. 02, p. 4-20, 2014.

BEZERRA, Joel Medeiros; SILVA, Paulo Cesar Moura; BATISTA, Rafael Oliveira; PINTO, Carlos Henrique Catunda; FEITOSA, Alex Pinheiro. Análise dos indicadores de qualidade da água no trecho urbano do Rio Apodi-Mossoró em Mossoró-RN, Brasil. Semina: Ciências Agrárias, Londrina, v. 34, n. 6, p. 3443-3454, 2013.

BRASIL, Ministério do Meio Ambiente. Conselho Nacional Do Meio Ambiente (CONAMA). Resolução no 357 de 17 de março de 2005. Coleção de leis da República Federativa do Brasil. Brasília. DF, 23 p., 2005.

BRASIL. Fundação Nacional de Saúde. Manual prático de análise de água. 2 ed. Brasília: Fundação Nacional de Saúde. Saneamento I. 146 p, 2006. 
BRASIL. Ministério do Meio Ambiente. Decreto n 73.030 de 30 de outubro de 1973. Coleção de leis do Brasil. Brasília. DF, v. 8, 160 p., 1973.

BRASIL. Portaria do Ministério da Saúde № 2.914 de 12 de dezembro de 2011. Dispõe sobre os procedimentos de controle e de vigilância da qualidade da água para consumo humano e seu padrão de potabilidade. Disponível em:

http://site.sabesp.com.br/site/uploads/file/asabesp doctos/PortariaMS291412122011.pdf> Acesso em: 20 ago. 2016.

CERQUEIRA, Rui; BRANT, Arthur; NASCIMENTO, Marcelo Trindade; PARDINI, Renata. Fragmentação: alguns conceitos. Fragmentação de Ecossistemas: Causas. efeitos sobre a biodiversidade e recomendações de políticas públicas, Biodiversidade 6, Brasília: Ministério do Meio Ambiente, p. 24-40, 2003.

CHANG, Heejun. Spatial analysis of water quality trends in the Han River basin. South Korea, Water Research, v. 42, n. 13, p. 3285-3304, julho 2008.

DANELON, Jean Roger Bombonatto; NETTO, Fausto Miguel Luz; RODRIGUES, Silvio Carlos. Análise do nível de fósforo total, nitrogênio amoniacal e cloretos nas águas do córrego terra branca no município de Uberlândia (MG), Revista Geonorte, v. 1, n. 4, p. 412-421, 2012.

EMBRAPA. Boletim Climatológico Mensal. Disponível em: <http://agromet.cpact.embrapa.br/online/reports/junho2016.pdf>. Acesso em: 13 julho, 2016.

FERREIRA, Arlon Cândido; ROCHA, Leandro Cristian; FIGUEIREDO, Múcio Amaral. Análise do índice de qualidade de água na bacia do córrego do rio acima. São João Del-Rei/MG, Revista Nacional de Gerenciamento de Cidades, v. 3, n. 15, p. 94-105, 2015.

GOMES SILVA, Paulo Alexandre Jesus; LIMA, Suzy Darley; GOLIN, Rossean; FIGUEIREDO, Daniela Maimoni; LIMA, Zoraidy Marques; MORAIS, Eduardo Beraldo; DORES, Eliana Freire Gaspar Carvalho. Qualidade da água de uma micro bacia com fins de abastecimento público. Chapada dos Guimarães, MT, HOLOS, v. 4, n. 1, p. 22-33, 2014.

HANSMANN, Henrique Zanotta. Descrição e Caracterização das Principais Enchentes e Alagamentos de Pelotas-RS, Pelotas: UFPel, 2013. Monografia, Curso de Engenharia Ambiental e Sanitária, Universidade Federal de Pelotas, p. 63, 2013.

KUZMANOVIC' , Maja; DOVAL, Julio López; CATALA, Núria Castro; GUASCH, Helena; PETROVIC', Mira; MUÑOZ, Isabel; GINEBREDA, Antoni; BARCELÓ, Damià. Ecotoxicological risk assessment of chemical pollution in four Iberian river basins and its relationship with the aquatic macroinvertebrate community status, Science of the Total Environment, v. 540, n. 1, p. 324-333, jan. 2016.

MMA. Ministério do Meio Ambiente. Mapa de áreas prioritárias para conservação, uso sustentável e repartição de benefícios da biodiversidade brasileira. Disponível em:<http://www.mma.gov.br/images/arquivo/80049/mapas/AreasPrioritarias Brasil.jpg>. Acesso em: 29 de julho 2016.

MOHAMED, Ibrahim; OTHMANN, Farigah; IBRAHIM, Adriana; ELDIN, Alaa; YUNUS, Rossita. Assessment of water quality parameters using multivariate analysis for Klang River basin. Malaysia, Environmental Monitoring and Assessment, v. 187, p. 4182-4193, jan. 2015.

MULLER, Thais.; GRANADA, Camille Eichelberger; SPEROTTO, Raul Antonio. Qualidade da água de três locais com potenciais fontes de contaminação no Rio Taquari, RS, Revista Ambiente \& Água, v. 11 , n. 1 , p. 75-81, 2016. 
MWANAMOKI, Paola; DEVARAJAN, Naresh; THEVENON, Florian; ATIBU, Emmanuel; TSHIBANDA, Joseph; NGELINKOTO, Patience; MPIANA, Pius; PRABAKAR, Kandasamy; MUBEDI, Josué; KABELE, Chirstophe; WILDI, Walter; POTÉ John. Assessment of pathogenic bacteria in water and sediment from a water reservoir under tropical conditions (Lake Ma Vallée), Kinshasa Democratic Republic of Congo, Environmental Monitoring and Assessment, v. 186, n. 10, p. 6821-6830, out. 2014.

NASCIMENTO, Bruno Lucio Meneses; GOMES, Dianna Rayla Carneiro Souza; COSTA, Gleison Pereira; ARAÚJO, Suely Silva; SANTOS, Luiz Carlos Araújo; OLIVEIRA, Jorge Diniz. Comportamento e avaliação de metais potencialmente tóxicos (Cu (II). Cr (III). Pb (II) e Fe (III)) em águas superficiais dos Riachos Capivara e Bacuri Imperatriz-MA, Engenharia Sanitária e Ambiental, v. 20, n. 3, p. 369378, set. 2015.

NETO, Hélio de Paiva Brettas. Caracterização ambiental e determinação de parâmetros físicoquímicos. biológicos e índice de fragilidade ambiental da Micro Bacia Arroio Moreira / Fragata, Pelotas: UCPel, 2009. Monografia, Licenciatura em Ciências Biológicas, Universidade Católica de Pelotas, 2009.

NETTO, Antenor Oliveira Aguaiar; GARCIA, Alexandre Borges; ALVES, José Patrocínio Hora; FERREIRA, Robérto Anastácio; SILVA, Marinoé Gonzaga. Physical and chemical characteristics of water from the hydrographic basin of the Poxim River. Sergipe State. Brazil, Environmental Monitoring and Assessment, v. 185, p. 4417-4426, out. 2012.

PELOTAS, Prefeitura Municipal de Pelotas, Secretária de Qualidade Ambiental (SQA). Plano Ambiental de Pelotas, Pelotas, 120 p, 2013. Disponível em: < http://www.pelotas.rs.gov.br/qualidade-ambiental/plano-municipal/arquivos/Plano-Ambiental-dePelotas.pdf>. Acesso em: 09 de julho 2017.

PEREIRA, Régis Silva. Identificação e Caracterização das fontes de poluição em sistemas hídricos, Revista Eletrônica de Recursos Hídricos, IPH -UFRGS, v. 1, n. 1, p. 20-36, 2004.

ROCHA, Monyque Palagano; DOURADO, Priscila Leocadia Rosa; RODRIGUES, Mayara Souza; RAPOSO JUNIOR, Jorge Luiz; GRISOLIA, Alexeia Barufatti; OLIVEIRA, Kelly Mari, Pires. The influence of industrial and agricultural waste on water quality in the Água Boa stream (Dourados. Mato Grosso do Sul. Brazil), Environmental Monitoring and Assessment, v. 187, n. 7, p. 442-453, jul. 2015.

SANEP, Serviço autônomo de Saneamento de Pelotas. Captação, Disponível em: <http://www.pelotas.rs.gov.br/sanep/captacao/>. Acesso em: 15 de junho 2016.

SANTI, Giuliana Marconati; FURTADO, Cydia de Menezes; MENEZES, Rui Sant'Ana; KEPPELER, Erlei Cassiano. Variabilidade espacial de parâmetros e indicadores de qualidade da água na sub-bacia hidrográfica do Igarapés São Francisco, Rio Branco, Acre, Brasil, Ecologia Aplicada, v. 11, n. 1, p. 23-31, maio 2012.

SARAIVA, Vanessa Kelly; NASCIMENTO, Marcos Roberto Lopes; PALMIERI, Helena Eugênia Leonhardt; JACOMINO, Vanusa Maria Feliciano. Avaliação da qualidade de sedimentos - estudo de caso: subbacia do Ribeirão Espírito Santo, afluente do Rio São Francisco, Química Nova, v. 32, n. 8, p. 19952002, 2009.

SILVA, Gilmar Silvério; SANTOS, Edmilson Arruda; CORRÊA, Luciana Barbosa; MARQUES, Aldalea Lopes Brandes; MARQUES, Edmar Pereira; SOUSA, Eliane Rodrigues; SILVA, Gilberto Silvério. Avaliação integrada da qualidade de águas superficiais: grau de trofia e proteção da vida aquática nos rios Anil e Bacanga. São Luís (MA), Engenharia Sanitária e Ambiental, v. 19, n. 3, p. 245251, 2014. 
SILVA, Maria Socorro Ferreira; SOUZA, Rosemeri Melo. Padrões espaciais de fragmentação florestal na flona do Ibura - Sergipe, Mercator, v. 13, n. 3, p. 121-137, dez. 2014.

SOUZA, Marielle Medeiros; GASTALDINI, Maria do Carmo Cauduro. Avaliação da qualidade da água em bacias hidrográficas com diferentes impactos antrópicos, Engenharia Sanitária e Ambiental, v. 19, n. 3, p. 263-274, set. 2014.

TABARI, Hossein; MAROFI, Safar; AHMADI, Mohammad. Long-term variations of water quality parameters in the Maroon River, Iran, Environmental Monitoring and Assessment, v. 177. p. 273-287, 2011.

VALADÃO, Lidiane Schmalfuss; GARCIA, John Felipe; SANCHES FILHO, Pedro José. Caracterização físico-química da água do canal São Gonçalo. Pelotas/RS. XXXIII Congresso Interamercano de Engenharia Sanitária e Ambiental - ABES, Salvador/Ba: AIDIS, v. IV, p. 1-5, 2012. 